\title{
Guest Editorial: Variational Models, Convex Analysis and Numerical Optimization in Mathematical Imaging
}

\author{
Antonin Chambolle • Michael Hintermüller • \\ Thomas Pock • Christoph Schnörr • Gabriele Steidl
}

Published online: 10 August 2013

(C) Springer Science+Business Media New York 2013

Variational models in connection with convex analysis, relaxation methods, problem decomposition and numerical optimization pervade current research activities in mathematical imaging and vision. This special issue contains a series of papers presenting corresponding progress.

The intricate problem of jointly estimating stereo disparity and illumination variation is considered in the paper "A Parallel Proximal Splitting Method for Disparity Estimation from Multicomponent Images Under Illumination Variation" (doi:10.1007/s10851-012-0361-z). This initially non-convex problem is tackled by a first order Taylor approximation and solving a sequence of convex problems. Additionally, the paper demonstrates the application of an advanced decomposition technique that enables the parallel implementation of the numerical optimization algorithm on GPUs.

Authors of the paper "A Variational Framework for Region-Based Segmentation Incorporating Physical Noise

A. Chambolle

CMAP, Ecole Polytechnique, Palaiseau, France

e-mail: antonin.chambolle@cmap.polytechnique.fr

M. Hintermüller

Humboldt-University of Berlin, Berlin, Germany

e-mail: hint@math.hu-berlin.de

T. Pock

Graz University of Technology, Graz, Austria

e-mail: pock@icg.tugraz.at

C. Schnörr $(\varangle)$

Heidelberg University, Heidelberg, Germany

e-mail: schnoerr@math.uni-heidelberg.de

G. Steidl

Technical University of Kaiserslautern, Kaiserlautern, Germany

e-mail: steidl@mathematik.uni-kl.de
Models" (doi:10.1007/s10851-013-0419-6) rigorously investigate from a continuous variational perspective various probabilistic models of local data likelihood, beyond the basic Gaussian assumption. These models are relevant to a range a practical imaging scenarios. An alternating numerical scheme for model evaluation and model parameter estimation is devised as well.

A connection between convex lower level feasible sets constraining a convex objective function and the regularization parameter of the corresponding penalty formulation is worked out in the paper "Homogeneous Penalizers and Constraints in Convex Image Restoration" (doi:10.1007/ s10851-012-0392-5). The problem class considered encompasses a broad range of variational approaches to image processing of current interest and also makes a connection to the problem of tracing the regularization path in machine learning.

In the paper "Prox-Regularity of Rank Constraint Sets and Implications for Algorithms" (doi:10.1007/s10851-0120406-3), the prox-regularity of lower level sets of the rank function is established. This result enables the direct application of basic iterative, locally converging algorithms to rank constrained problems. It covers a significant portion of recovery problems of current research, without the need to resort to convex relaxations or heuristics.

A priori performance bounds for the continuous formulation of the variational image labeling (partitioning) problem are established in the paper "Optimality Bounds for a Variational Relaxation of the Image Partitioning Problem" (doi:10.1007/s10851-012-0390-7), for a broad class of regularizers. In particular, the probabilistic approach to convert optima of convex relaxations to high-quality combinatorial solutions is considered. Corresponding image partitions better preserve geometric image structure due to the underlying continuous formulation. 
Regularization of signals with periodic values, related to orientation and angles, phase, etc., is studied in the paper "Total Cyclic Variation and Generalizations" (doi:10.1007/ s10851-012-0396-1). Specifically, a natural total variation measure on the 1D torus is devised that is shift invariant and thus avoids commonly encountered wrap-around artefacts. Convex techniques of relaxation and optimization enable the efficient application of the approach to problem class.

Authors of the paper "Convex relaxation of a class of vertex penalizing functionals" (doi:10.1007/s10851-012- 0347-x) explore the functional lifting approach in connection with curvature based regularization, a topic with links to earlier work in many fields, ranging from visual perception to PDE-based and variational models. In particular, the paper provides a striking example for the sophisticated reformulation of an apparently intractable problem class within a convex variational relaxation framework.

The editors of this issue hope that readers of JMIV will enjoy these contributions to mathematical imaging and vision. 